\title{
Inequalities for Convex Functions on Simplexes and Their Cones
}

\author{
Zlatko Pavić ${ }^{1}$ and Shanhe $\mathrm{Wu}^{2}$ \\ ${ }^{1}$ Mechanical Engineering Faculty in Slavonski Brod, University of Osijek, Trg Ivane Brlić Mažuranić 2, 35000 Slavonski Brod, Croatia \\ ${ }^{2}$ Department of Mathematics and Computer Science, Longyan University, Longyan, Fujian 364012, China
}

Correspondence should be addressed to Shanhe Wu; shanhewu@gmail.com

Received 9 April 2014; Accepted 17 July 2014; Published 27 October 2014

Academic Editor: Vladimir Georgiev

Copyright (C) 2014 Z. Pavić and S. Wu. This is an open access article distributed under the Creative Commons Attribution License, which permits unrestricted use, distribution, and reproduction in any medium, provided the original work is properly cited.

The aim of this paper is to present the fundamental inequalities for convex functions on Euclidean spaces. The work is based on the geometry of the simplest convex sets and properties of convex functions. Some obtained inequalities are applied to demonstrate a natural way of generalizing the Hermite-Hadamard inequality.

\section{Introduction}

Let $\mathscr{X}$ be a real linear (vector) space, $a_{i} \in \mathscr{X}$ points (vectors), and $\alpha_{i} \in \mathbb{R}$ coefficients (scalars). The combination

$$
c=\sum_{i=1}^{n} \alpha_{i} a_{i}
$$

belongs to the linear subspace $\mathscr{L}=\operatorname{lin}\left\{a_{i}\right\}$ as the smallest linear space that contains all $a_{i}$, and it is called the linear combination. If $\sum_{i=1}^{n} \alpha_{i}=1$, the combination in (1) belongs to the affine hull $\mathscr{A}=\operatorname{aff}\left\{a_{i}\right\}$ as the smallest translated linear space that contains all $a_{i}$, and it is called the affine combination. If $\sum_{i=1}^{n} \alpha_{i}=1$ and all $\alpha_{i} \in[0,1]$, the combination in (1) belongs to the convex hull $\mathscr{C}=\operatorname{conv}\left\{a_{i}\right\}$ as the smallest convex point set that contains all $a_{i}$, and it is called the convex combination. The point $c$ itself is called the center of the observed combination.

A function $f: \mathscr{X} \rightarrow \mathbb{R}$ is affine if the equality

$$
f(c)=\sum_{i=1}^{n} \alpha_{i} f\left(a_{i}\right)
$$

holds for all affine combinations in (1), and $f$ is convex if the inequality

$$
f(c) \leq \sum_{i=1}^{n} \alpha_{i} f\left(a_{i}\right)
$$

holds for all convex combinations in (1). The formulas may be referred to for $n=2$ and then by applying the mathematical induction extended to any positive integer $n$. The important Jensen's inequality in (3) was just so proven in [1].

Jensen's inequality was extended to special affine combinations and their centers in [2], generalizing Mercer's variant of Jensen's inequality obtained in [3]. The connection between discrete and integral forms was observed in [4]; the integral forms were studied in [5], and the Jensen type inequalities for Q-class functions were investigated in [6]. Different variants and forms can be found in $[7,8]$.

\section{Convex Functions on the Line}

Known results are presented in this section, in a way that can be generalized. Convex combinations of the line segment and affine combinations of the convex cone represent the backbone of the work. Theorem 3 is the most important in terms of scope of its content.

If $a, b \in \mathbb{R}$ are different numbers, then every number $x \in$ $\mathbb{R}$ can be uniquely presented as the affine combination

$$
x=\alpha a+\beta b,
$$

where

$$
\alpha=\frac{\left|\begin{array}{ll}
x & 1 \\
b & 1
\end{array}\right|}{\left|\begin{array}{ll}
a & 1 \\
b & 1
\end{array}\right|}, \quad \beta=-\frac{\left|\begin{array}{ll}
x & 1 \\
a & 1
\end{array}\right|}{\left|\begin{array}{ll}
a & 1 \\
b & 1
\end{array}\right|} .
$$

The above binomial combination is convex if and only if the number $x$ belongs to the interval $\operatorname{conv}\{a, b\}$. Given the function $f: \mathbb{R} \rightarrow \mathbb{R}$, let $f_{\{a, b\}}^{\text {line }}: \mathbb{R} \rightarrow \mathbb{R}$ be the function of 
the line passing through the points $(a, f(a))$ and $(b, f(b))$ of the graph of $f$. Using the affinity of $f_{\{a, b\}}^{\text {line }}$, we get

$$
f_{\{a, b\}}^{\text {line }}(x)=\alpha f(a)+\beta f(b) .
$$

Let $\mathscr{C}_{a}$ be the convex cone (half-line) with the vertex at $a$ spanned by $a-b$ containing binomial affine combinations $x=a+p(a-b)=(1+p) a-p b$, where $p \geq 0$; that is,

$$
\mathscr{C}_{a}=\{(1+p) a-p b: p \geq 0\} .
$$

Convex cone $\mathscr{C}_{b}$ is defined similarly. That means that if $a<b$, then $\mathscr{C}_{a}=(-\infty, a]$ and $\mathscr{C}_{b}=[b,+\infty)$. The consequence of the representations in (4) and (6) is the well-known characterization of convex functions with one variable, as specified in the following lemma.

Lemma 1. If $a, b \in \mathbb{R}$ are the line segment endpoints, then every convex function $f: \mathbb{R} \rightarrow \mathbb{R}$ satisfies the inequality

$$
f(x) \leq f_{\{a, b\}}^{\text {line }}(x) \quad \text { for } x \in \operatorname{conv}\{a, b\}
$$

and the reverse inequality

$$
f(x) \geq f_{\{a, b\}}^{\text {line }}(x) \quad \text { for } x \in \mathscr{C}_{a} \cup \mathscr{C}_{b} .
$$

Using combinations $x=(1+p) a-p b$ with $p \geq 0$, the inequality in (9) takes the form

$$
f((1+p) a-p b) \geq(1+p) f(a)-p f(b) .
$$

Example 2. Let $a, b$ and $x=(1+p) a-p b \in \mathscr{C}_{a}$ be positive real numbers.

Substituting the values of the convex power function $f(x)=x^{r}$ with the exponent $r \in(-\infty, 0] \cup[1,+\infty)$ in the inequality in (10), we get the inequality

$$
[(1+p) a-p b]^{r} \geq(1+p) a^{r}-p b^{r}
$$

and the reverse inequality by substituting the values of the concave power function with the exponent $r \in[0,1]$.

Substituting the values of the concave logarithmic function $f(x)=\ln x$ in the inequality in (10) and rearranging them, it follows the inequality

$$
a^{1+p} b^{-p} \geq(1+p) a-p b \text {. }
$$

Combining the application of Lemma 1 and Jensen's inequality to the convex combinations $x=\sum_{i=1}^{n} \alpha_{i} a_{i}$, it follows

$$
f(x) \leq \sum_{i=1}^{n} \alpha_{i} f\left(a_{i}\right) \leq f_{\{a, b\}}^{\text {line }}(x)
$$

if all $a_{i} \in \operatorname{conv}\{a, b\}$ and

$$
\sum_{i=1}^{n} \alpha_{i} f\left(a_{i}\right) \geq f(x) \geq f_{\{a, b\}}^{\text {line }}(x)
$$

if all $a_{i} \in \mathscr{C}_{a}$ or all $a_{i} \in \mathscr{C}_{b}$.

Convex combinations with the common center are considered in the following theorem.
Theorem 3. Let $a, b \in \mathbb{R}$ be the line segment endpoints. Let $\sum_{i=1}^{n} \alpha_{i} a_{i}$ be a convex combination of the points $a_{i} \in$ conv $\{a, b\}$, and let $\sum_{j=1}^{m} \beta_{j} b_{j}$ be a convex combination of the points $b_{j} \in \mathscr{C}_{a} \cup \mathscr{C}_{b}$.

If the center equality

$$
c=\sum_{i=1}^{n} \alpha_{i} a_{i}=\sum_{j=1}^{m} \beta_{j} b_{j}
$$

is valid, then the inequality

$$
f(c) \leq \sum_{i=1}^{n} \alpha_{i} f\left(a_{i}\right) \leq \sum_{j=1}^{m} \beta_{j} f\left(b_{j}\right)
$$

holds for every convex function $f: \mathbb{R} \rightarrow \mathbb{R}$.

Proof. The first inequality in (16) is the Jensen inequality. The last inequality follows from the series of inequalities

$$
\begin{aligned}
\sum_{i=1}^{n} \alpha_{i} f\left(a_{i}\right) & \leq \sum_{i=1}^{n} \alpha_{i} f_{\{a, b\}}^{\text {line }}\left(a_{i}\right)=f_{\{a, b\}}^{\text {line }}\left(\sum_{i=1}^{n} \alpha_{i} a_{i}\right) \\
& =f_{\{a, b\}}^{\text {line }}\left(\sum_{j=1}^{m} \beta_{j} b_{j}\right)=\sum_{j=1}^{m} \beta_{j} f_{\{a, b\}}^{\text {line }}\left(b_{j}\right) \\
& \leq \sum_{j=1}^{m} \beta_{j} f\left(b_{j}\right)
\end{aligned}
$$

derived by applying the inequality in (8) to $a_{i}$ and the inequality in (9) to $b_{j}$.

The geometric insight to the inequality in (16) presented in Figure 1 shows that the point $P_{1}(c, f(c))$ is below the point

$$
P_{2}\left(c, \sum_{i=1}^{n} \alpha_{i} f\left(a_{i}\right)\right) \in \operatorname{conv}\left\{\left(a_{1}, f\left(a_{1}\right)\right), \ldots,\left(a_{n}, f\left(a_{n}\right)\right)\right\},
$$

and the point $P_{2}$ is below the point

$$
P_{3}\left(c, \sum_{j=1}^{m} \beta_{j} f\left(b_{j}\right)\right) \in \operatorname{conv}\left\{\left(b_{1}, f\left(b_{1}\right)\right), \ldots,\left(b_{m}, f\left(b_{m}\right)\right)\right\} .
$$

Corollary 4. Let $a, b \in \mathbb{R}$ be the line segment endpoints. Let $\sum_{i=1}^{n} \alpha_{i} a_{i}$ be a convex combination of the points $a_{i} \in$ conv $\{a, b\}$, and let $\alpha a+\beta b$ be the convex combination such that

$$
c=\sum_{i=1}^{n} \alpha_{i} a_{i}=\alpha a+\beta b
$$

Then the inequality

$$
f(c) \leq \sum_{i=1}^{n} \alpha_{i} f\left(a_{i}\right) \leq \alpha f(a)+\beta f(b)
$$

holds for every convex function $f: \operatorname{conv}\{a, b\} \rightarrow \mathbb{R}$. 


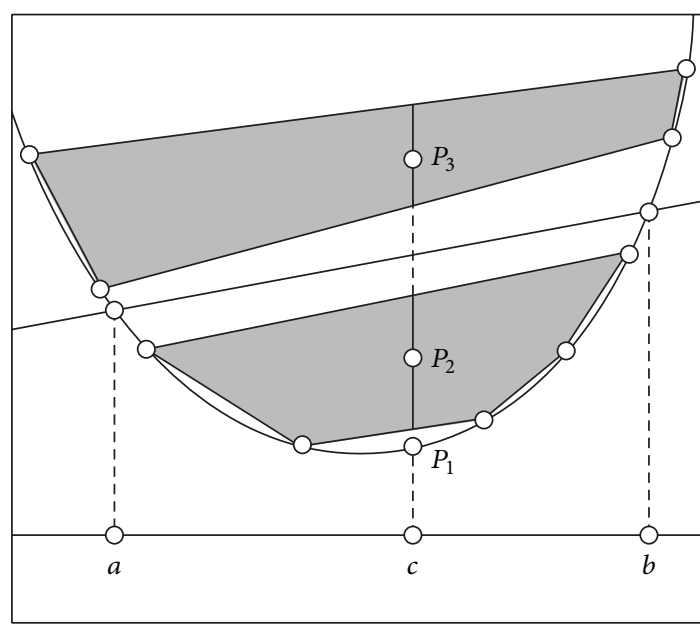

FIGURE 1: Geometric representation of the inequality in (16).

\section{Main Results: Convex Functions on the Plane}

We assume that $\mathbb{R}^{2}$ is the real vector space with the standard coordinate addition $\left(x_{1}, y_{1}\right)+\left(x_{2}, y_{2}\right)=\left(x_{1}+x_{2}, y_{1}+y_{2}\right)$ and the scalar multiplication $\alpha(x, y)=(\alpha x, \alpha y)$.

If $A\left(x_{A}, y_{A}\right), B\left(x_{B}, y_{B}\right)$, and $C\left(x_{C}, y_{C}\right)$ are the planar points that do not belong to one line, then every point $P(x, y) \in \mathbb{R}^{2}$ can be presented by the unique affine combination:

$$
P=\alpha A+\beta B+\gamma C
$$

where

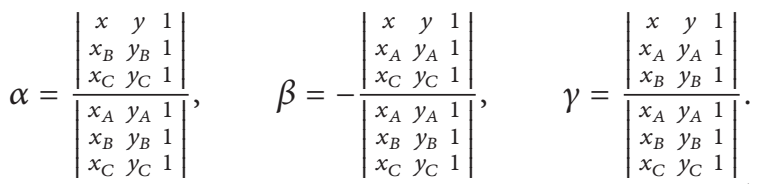

The above trinomial combination is convex if and only if point $P$ belongs to the triangle $\operatorname{conv}\{A, B, C\}$. Given the function $f: \mathbb{R}^{2} \rightarrow \mathbb{R}$, let $f_{\{A, B, C\}}^{\text {plane }}: \mathbb{R}^{2} \rightarrow \mathbb{R}$ be the function of the plane passing through the points $(A, f(A)),(B, f(B))$, and $(C, f(C))$ of the graph of $f$. Because of the affinity of $f_{\{A, B, C\}}^{\text {plane }}$, it follows

$$
f_{\{A, B, C\}}^{\text {plane }}(P)=\alpha f(A)+\beta f(B)+\gamma f(C) .
$$

Let $\mathscr{C}_{A}$ be the convex cone with the vertex at $A$ spanned by the vectors $A-B$ and $A-C$ containing trinomial affine combinations $P=A+p(A-B)+q(A-C)=(1+p+q) A-$ $p B-q C$, where $p, q \geq 0$; that is,

$$
\mathscr{C}_{A}=\{(1+p+q) A-p B-q C: p, q \geq 0\} .
$$

Cones $\mathscr{C}_{B}$ and $\mathscr{C}_{C}$ are defined in the same way, and all tree cones can be seen in Figure 2.

Using the cones, we achieve the generalization of Lemma 1 to convex functions on the plane as follows.

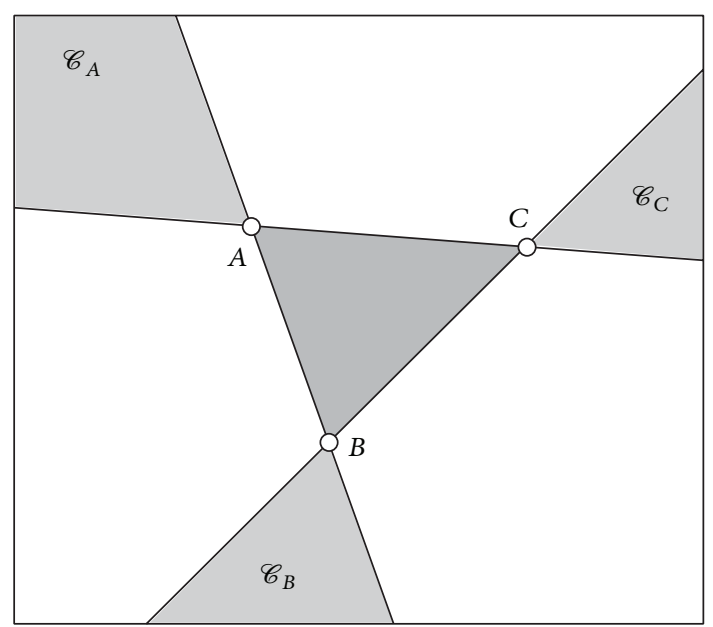

FIgURE 2: Triangle with cones.

Lemma 5. If $A, B, C \in \mathbb{R}^{2}$ are the triangle vertices, then every convex function $f: \mathbb{R}^{2} \rightarrow \mathbb{R}$ satisfies the inequality

$$
f(P) \leq f_{\{A, B, C\}}^{\text {plane }}(P) \quad \text { for } P \in \operatorname{conv}\{A, B, C\},
$$

and the reverse inequality

$$
f(P) \geq f_{\{A, B, C\}}^{\text {plane }}(P) \quad \text { for } P \in \mathscr{C}_{A} \cup \mathscr{C}_{B} \cup \mathscr{C}_{C} .
$$

Proof. If $P \in \operatorname{conv}\{A, B, C\}$, the combination in (22) is convex, and the inequality in (26) follows from the convexity of $f$ and the equality in (24).

If $P \in \mathscr{C}_{A} \cup \mathscr{C}_{B} \cup \mathscr{C}_{C}$, say $P \in \mathscr{C}_{A}$ and $P \neq A$, we can represent the point $P$ as the binomial affine combination:

$$
\begin{aligned}
P & =(1+p+q) A-p B-q C \\
& =(1+p+q) A-(p+q)\left(\frac{p}{p+q} B+\frac{q}{p+q} C\right) .
\end{aligned}
$$

Applying the inequality in (10) to the combination in (29), then using the convexity of $f$ and the affinity of $f_{\{A, B, C\}}^{\text {plane }}$, we get the series of inequalities:

$$
\begin{aligned}
f(P) & \geq(1+p+q) f(A)-(p+q) f\left(\frac{p}{p+q} B+\frac{q}{p+q} C\right) \\
& \geq(1+p+q) f(A)-p f(B)-q f(C) \\
& =f_{\{A, B, C\}}^{\text {plane }}((1+p+q) A-p B-q C)=f_{\{A, B, C\}}^{\text {plane }}(P)
\end{aligned}
$$

which includes the inequality in (27).

The area outside the triangle and outside the cones (the white area in Figure 2) cannot be generally covered with one inequality. Such area does not exist in the previous onedimensional case.

Applying Lemma 5 and Jensen's inequality to the planar convex combinations $P=\sum_{i=1}^{n} \alpha_{i} A_{i}$, we get the inequality

$$
f(P) \leq \sum_{i=1}^{n} \alpha_{i} f\left(A_{i}\right) \leq f_{\{A, B, C\}}^{\text {plane }}(P)
$$


if all $A_{i} \in \operatorname{conv}\{A, B, C\}$ and the inequality

$$
\sum_{i=1}^{n} \alpha_{i} f\left(A_{i}\right) \geq f(P) \geq f_{\{A, B, C\}}^{\text {plane }}(P)
$$

if all $A_{i} \in \mathscr{C}_{A}$, or all $A_{i} \in \mathscr{C}_{B}$, or all $A_{i} \in \mathscr{C}_{C}$.

The following are planar convex combinations with the common center.

Theorem 6. Let $A, B, C \in \mathbb{R}^{2}$ be the triangle vertices. Let $\sum_{i=1}^{n} \alpha_{i} A_{i}$ be a convex combination of the points $A_{i} \in$ conv $\{A, B, C\}$, and let $\sum_{j=1}^{m} \beta_{j} B_{j}$ be a convex combination of the points $B_{j} \in \mathscr{C}_{A} \cup \mathscr{C}_{B} \cup \mathscr{C}_{C}$.

If the center equality

$$
P=\sum_{i=1}^{n} \alpha_{i} A_{i}=\sum_{j=1}^{m} \beta_{j} B_{j}
$$

is valid, then the inequality

$$
f(P) \leq \sum_{i=1}^{n} \alpha_{i} f\left(A_{i}\right) \leq \sum_{j=1}^{m} \beta_{j} f\left(B_{j}\right)
$$

holds for every convex function $f: \mathbb{R}^{2} \rightarrow \mathbb{R}$.

Proof. We employ the proof of Theorem 3 using $f_{\{A, B, C\}}^{\text {plane }}$ instead of $f_{\{a, b\}}^{\text {line }}$.

If (33) is valid, and if all points $A_{i}$ and $B_{j}$ are the triangle vertices, then the equality

$$
\sum_{i=1}^{n} \alpha_{i} f\left(A_{i}\right)=\sum_{j=1}^{m} \beta_{j} f\left(B_{j}\right)
$$

holds. The reverse statement is true if the function $f$ is strictly convex.

Corollary 7. Let $A, B, C \in \mathbb{R}^{2}$ be the triangle vertices. Let $\sum_{i=1}^{n} \alpha_{i} A_{i}$ be a convex combination of the points $A_{i} \in$ conv $\{A, B, C\}$, and let $\alpha A+\beta B+\gamma C$ be the convex combination such that

$$
P=\sum_{i=1}^{n} \alpha_{i} A_{i}=\alpha A+\beta B+\gamma C .
$$

Then the inequality

$$
f(P) \leq \sum_{i=1}^{n} \alpha_{i} f\left(A_{i}\right) \leq \alpha f(A)+\beta f(B)+\gamma f(C)
$$

holds for every convex function $f: \operatorname{conv}\{A, B, C\} \rightarrow \mathbb{R}$.

\section{Generalization to Higher Dimensions}

Let $P_{1}, \ldots, P_{N+1} \in \mathbb{R}^{N}$ be the points so that the vectors $P_{1}-P_{2}, \ldots, P_{1}-P_{N+1}$ be linearly independent. In this case, the convex hull conv $\left\{P_{1}, \ldots, P_{N+1}\right\}$ is called the $N$-simplex in $\mathbb{R}^{N}$ with the vertices $P_{1}, \ldots, P_{N+1}$. All of the simplex vertices cannot belong to the same hyperplane in $\mathbb{R}^{N}$. Every point $P \in \mathbb{R}^{N}$ can be presented by the unique affine combination:

$$
P=\sum_{k=1}^{N+1} \alpha_{k} P_{k}
$$

where the coefficients

$$
\alpha_{k}=(-1)^{k+1} \frac{\left|\begin{array}{cccc}
x_{1} & \cdots & x_{N} & 1 \\
x_{11} & \cdots & x_{1 N} & 1 \\
\vdots & \ddots & \vdots & \vdots \\
x_{k-1} & \cdots & x_{k-1 N} & 1 \\
x_{k+1} & \cdots & x_{k+1 N} & 1 \\
\vdots & \ddots & \vdots & \vdots \\
x_{N+1} & \cdots & x_{N+1 N} & 1
\end{array}\right|}{\left|\begin{array}{cccc}
x_{11} & \cdots & x_{1 N} & 1 \\
\vdots & \ddots & \vdots & \vdots \\
x_{k-1} & \cdots & x_{k-1 N} & 1 \\
x_{k 1} & \cdots & x_{k N} & 1 \\
x_{k+1} & \cdots & x_{k+1 N} & 1 \\
\vdots & \ddots & \vdots & \vdots \\
x_{N+1} & \cdots & x_{N+1 N} & 1
\end{array}\right|}
$$

are determined generalizing the coefficients in (23). The combination in (38) is convex if and only if the point $P$ belongs to the $N$-simplex $\operatorname{conv}\left\{P_{1}, \ldots, P_{N+1}\right\}$.

Given the function $f: \mathbb{R}^{N} \rightarrow \mathbb{R}$, let $f_{\left\{P_{1}, \ldots, P_{N+1}\right\}}^{\text {hyperplane }}:$ $\mathbb{R}^{N} \rightarrow \mathbb{R}$ be the function of the hyperplane (in $\mathbb{R}^{N+1}$ ) passing through the points $\left(P_{k}, f\left(P_{k}\right)\right)$ of the graph of $f$. Therefore

$$
f_{\left\{P_{1}, \ldots, P_{N+1}\right\}}^{\text {hyperplane }}(P)=\sum_{k=1}^{N+1} \alpha_{k} f\left(P_{k}\right) .
$$

Let $\mathscr{C}_{k}(k=1, \ldots, N+1)$ be the convex cone with the vertex at $P_{k}$ spanned by the vectors $P_{k}-P_{j}$ for $k \neq$ $j=1, \ldots, N+1$ containing $(N+1)$-membered affine combinations $P=P_{k}+\sum_{k \neq j=1}^{N+1} p_{j}\left(P_{k}-P_{j}\right)=\left(1+\sum_{k \neq j=1}^{N+1} p_{j}\right) P_{k}-$ $\sum_{k \neq j=1}^{N+1} p_{j} P_{j}$, where all $p_{j} \geq 0$; that is,

$$
\mathscr{C}_{k}=\left\{\left(1+\sum_{k \neq j=1}^{N+1} p_{j}\right) P_{k}-\sum_{k \neq j=1}^{N+1} p_{j} P_{j}: p_{j} \geq 0\right\} .
$$

Lemma 8. If $P_{1}, \ldots, P_{N+1} \in \mathbb{R}^{N}$ are the $N$-simplex vertices, then every convex function $f: \mathbb{R}^{N} \rightarrow \mathbb{R}$ satisfies the inequality

$$
f(P) \leq f_{\left\{P_{1}, \ldots, P_{N+1}\right\}}^{\text {hyperplane }}(P) \quad \text { for } P \in \operatorname{conv}\left\{P_{1}, \ldots, P_{N+1}\right\}
$$

and the reverse inequality

$$
f(P) \geq f_{\left\{P_{1}, \ldots, P_{N+1}\right\}}^{\text {hyperplane }}(P) \quad \text { for } P \in \mathscr{C}_{1} \cup \cdots \cup \mathscr{C}_{N+1} \text {. }
$$

Proof. The proof is similar to that of Lemma 5. We sketch the arguments briefly as follows.

To prove (42), we firstly apply Jensen's inequality to the convex combination $P=\sum_{k=1}^{N+1} \alpha_{k} P_{k} \in \operatorname{conv}\left\{P_{1}, \ldots, P_{N+1}\right\}$ and then use (40). 
To prove (43) for $P \in \mathscr{C}_{k}$ other than $P_{k}$, we firstly implement the inequality in (10) to the binomial affine combination:

$$
\begin{aligned}
P & =\left(1+\sum_{k \neq j=1}^{N+1} p_{j}\right) P_{k}-\sum_{k \neq j=1}^{N+1} p_{j} P_{j} \\
& =(1+p) P_{k}-p Q_{k},
\end{aligned}
$$

where $p=\sum_{k \neq j=1}^{N+1} p_{j}$ and $Q_{k}=\sum_{k \neq j=1}^{N+1}\left(p_{j} / p\right) P_{j}$ and then apply Jensen's inequality to the convex combination of $Q_{k}$, thus obtaining

$$
\begin{aligned}
f(P) & \geq\left(1+\sum_{k \neq j=1}^{N+1} p_{j}\right) f\left(P_{k}\right)-\sum_{k \neq j=1}^{N+1} p_{j} f\left(P_{j}\right) \\
& =f_{\left\{P_{1}, \ldots, P_{N+1}\right\}}^{\text {hyperplane }}(P)
\end{aligned}
$$

as the desired inequality.

Using the inequality in (45) with power and logarithmic functions, the inequalities of Example 2 can be generalized as follows.

Example 9. Let $P_{j}\left(x_{j 1}, \ldots, x_{j N}\right) \in \mathbb{R}^{N}$ for $j=1, \ldots, N+1$ be the $N$-simplex vertices with all coordinates $x_{j i}>0$. Let $P\left(x_{1}, \ldots, x_{N}\right) \in \mathscr{C}_{k}$ be a point with all coordinates $x_{i}=(1+$ p) $x_{k i}-\sum_{k \neq j=1}^{N+1} p_{j} x_{j i}>0$, where $p=\sum_{k \neq j=1}^{N+1} p_{j}$.

Including the values of the convex power sum function

$$
f\left(x_{1}, \ldots, x_{N}\right)=\sum_{i=1}^{N} x_{i}^{r_{i}} \quad \text { with all } r_{i} \in(-\infty, 0] \cup[1,+\infty)
$$

in the inequality in (45), we get the inequality

$$
\begin{array}{r}
\sum_{i=1}^{N}\left[(1+p) x_{k i}-\sum_{k \neq j=1}^{N+1} p_{j} x_{j i}\right]^{r_{i}} \\
\geq \sum_{i=1}^{N}\left[(1+p) x_{k i}^{r_{i}}-\sum_{k \neq j=1}^{N+1} p_{j} x_{j i}^{r_{i}}\right]
\end{array}
$$

and the reverse inequality by including the values of the concave power sum function with all $r_{i} \in[0,1]$.

Including the values of the concave logarithmic sum function

$$
f\left(x_{1}, \ldots, x_{N}\right)=\sum_{i=1}^{N} \ln x_{i}=\ln \prod_{i=1}^{N} x_{i}
$$

in the inequality in (45) and rearranging them, it follows the inequality

$$
\prod_{i=1}^{N}\left[x_{k i}^{1+p} \prod_{k \neq j=1}^{N+1} x_{j i}^{-p_{j}}\right] \geq \prod_{i=1}^{N}\left[(1+p) x_{k i}-\sum_{k \neq j=1}^{N+1} p_{j} x_{j i}\right] .
$$

Applying Lemma 8 and Jensen's inequality to the convex combinations $P=\sum_{i=1}^{n} \alpha_{i} A_{i}$, we get the inequality

$$
f(P) \leq \sum_{i=1}^{n} \alpha_{i} f\left(A_{i}\right) \leq f_{\left\{P_{1}, \ldots, P_{N+1}\right\}}^{\text {hyperplane }}(P)
$$

if all $A_{i} \in \operatorname{conv}\left\{P_{1}, \ldots, P_{N+1}\right\}$ and the inequality

$$
\sum_{i=1}^{n} \alpha_{i} f\left(A_{i}\right) \geq f(P) \geq f_{\left\{P_{1}, \ldots, P_{N+1}\right\}}^{\text {hyperplane }}(P)
$$

if all $A_{i}$ belong to the same cone $\mathscr{C}_{k}$.

Relying on Lemma 8, we reach the conclusion written in the next theorem.

Theorem 10. Let $P_{1}, \ldots, P_{N+1} \in \mathbb{R}^{N}$ be the $N$-simplex vertices. Let $\sum_{i=1}^{n} \alpha_{i} A_{i}$ be a convex combination of the points $A_{i} \in \operatorname{conv}\left\{P_{1}, \ldots, P_{N+1}\right\}$, and let $\sum_{j=1}^{m} \beta_{j} B_{j}$ be a convex combination of the points $B_{j} \in \mathscr{C}_{1} \cup \cdots \cup \mathscr{C}_{N+1}$.

If the center equality

$$
P=\sum_{i=1}^{n} \alpha_{i} A_{i}=\sum_{j=1}^{m} \beta_{j} B_{j}
$$

is valid, then the inequality

$$
f(P) \leq \sum_{i=1}^{n} \alpha_{i} f\left(A_{i}\right) \leq \sum_{j=1}^{m} \beta_{j} f\left(B_{j}\right)
$$

holds for every convex function $f: \mathbb{R}^{N} \rightarrow \mathbb{R}$.

Corollary 11. Let $P_{1}, \ldots, P_{N+1} \in \mathbb{R}^{N}$ be the $N$-simplex vertices. Let $\sum_{i=1}^{n} \alpha_{i} A_{i}$ be a convex combination of the points $A_{i} \in \operatorname{conv}\left\{P_{1}, \ldots, P_{N+1}\right\}$, and let $\sum_{j=1}^{N+1} \beta_{j} P_{j}$ be the convex combination such that

$$
P=\sum_{i=1}^{n} \alpha_{i} A_{i}=\sum_{j=1}^{N+1} \beta_{j} P_{j} .
$$

Then the inequality

$$
f(P) \leq \sum_{i=1}^{n} \alpha_{i} f\left(A_{i}\right) \leq \sum_{j=1}^{N+1} \beta_{j} f\left(P_{j}\right)
$$

holds for every convex function $f: \operatorname{conv}\left\{P_{1}, \ldots, P_{N+1}\right\} \rightarrow \mathbb{R}$.

\section{Application to the Hermite-Hadamard Inequality}

Applying the integral method with the convex combinations to the inequalities obtained in the theorems, we get their integral forms. Using the Jensen type inequalities, we briefly demonstrate the generalization of the Hermite-Hadamard inequality (for essentials on this inequality see [9] or [10]).

Let $f:[a, b] \rightarrow \mathbb{R}$ be a convex function. Given the positive integer $n$, we make the partition $[a, b]=\cup_{i=1}^{n}\left[a_{n i}, b_{n i}\right]$, where all subsegments have the same length $(b-a) / n$, and 
the adjacent subsegments have a common endpoint. If we take subsegment centers $x_{n i}=\left(a_{n i}+b_{n i}\right) / 2$, then we have the convex combination equality:

$$
c=\sum_{i=1}^{n} \frac{b_{n i}-a_{n i}}{b-a} x_{n i}=\frac{a+b}{2} .
$$

Applying the inequality in (21) to the above convex combination, it follows

$$
f\left(\frac{a+b}{2}\right) \leq \sum_{i=1}^{n} \frac{b_{n i}-a_{n i}}{b-a} f\left(x_{n i}\right) \leq \frac{f(a)+f(b)}{2},
$$

and letting $n$ to infinity, we obtain the classic HermiteHadamard inequality:

$$
f\left(\frac{a+b}{2}\right) \leq \frac{1}{b-a} \int_{[a, b]} f(x) d x \leq \frac{f(a)+f(b)}{2} .
$$

The transition to the planar case can be done using Corollary 7. Let $f: \triangle \rightarrow \mathbb{R}$ be a convex function, where $\triangle=\operatorname{conv}\{A, B, C\}$ is the triangle with vertices $A, B$, and $C$. Given the positive integer $n$, we use the partition $\triangle=\cup_{i=1}^{n^{2}} \triangle_{i}$ with congruent subtriangles $\triangle_{i}=\operatorname{conv}\left\{A_{n i}, B_{n i}, C_{n i}\right\}$ having a common edge or endpoint if they are adjacent. So, the area $\operatorname{ar}\left(\triangle_{i}\right)$ of each subtriangle $\triangle_{i}$ is equal to $\operatorname{ar}(\triangle) / n^{2}$. If we take subtriangle centers $P_{n i}=\left(A_{n i}+B_{n i}+C_{n i}\right) / 3$, then we have the convex combination equality:

$$
P=\sum_{i=1}^{n^{2}} \frac{\operatorname{ar}\left(\triangle_{i}\right)}{\operatorname{ar}(\triangle)} P_{n i}=\frac{A+B+C}{3} .
$$

Applying the inequality in (37) to the above convex combination, it follows

$$
f\left(\frac{A+B+C}{3}\right) \leq \sum_{i=1}^{n^{2}} \frac{\operatorname{ar}\left(\triangle_{i}\right)}{\operatorname{ar}(\triangle)} P_{n i} \leq \frac{f(A)+f(B)+f(C)}{3},
$$

and letting $n$ to infinity, we obtain the planar HermiteHadamard inequality:

$$
\begin{aligned}
& f\left(\frac{A+B+C}{3}\right) \\
& \quad \leq \frac{1}{\operatorname{ar}(\triangle)} \int_{\triangle} f(x, y) d x d y \leq \frac{f(A)+f(B)+f(C)}{3} .
\end{aligned}
$$

The transition to any dimension $N$ suggests Corollary 11 using the $N$-simplex $\triangle=\operatorname{conv}\left\{P_{1}, \ldots, P_{N+1}\right\}$. Applying the previous procedure to $\triangle$, we reach the conclusion that every convex function $f: \triangle \rightarrow \mathbb{R}$ satisfies the inequality

$$
\begin{aligned}
f\left(\frac{1}{N+1} \sum_{k=1}^{N+1} P_{k}\right) & \leq \frac{1}{\operatorname{vol}(\triangle)} \int_{\triangle} f\left(x_{1}, \ldots, x_{N}\right) d x_{1} \cdots d x_{N} \\
& \leq \frac{1}{N+1} \sum_{k=1}^{N+1} f\left(P_{k}\right) .
\end{aligned}
$$

\section{Conflict of Interests}

The authors declare that there is no conflict of interests regarding the publication of this paper.

\section{Acknowledgments}

The work of the first author has been fully supported by Mechanical Engineering Faculty in Slavonski Brod. The work of the second author has been supported by the Foundation of Scientific Research Project of Fujian Province Education Department of China under Grant JK2012049. The authors thank Velimir Pavić (graphic designer at Školska knjiga Zagreb) who has graphically prepared Figures 1 and 2.

\section{References}

[1] J. L. W. V. Jensen, "Om Konvekse Funktioner og Uligheder mellem Middelværdier," Nyt Tidsskrift for Matematik B, vol. 16, pp. 49-68, 1905.

[2] Z. Pavić, “The applications of functional variants of Jensen's inequality," Journal of Function Spaces and Applications, vol. 2013, Article ID 194830, 5 pages, 2013.

[3] A. M. Mercer, "A variant of Jensen's inequality, Journal of Inequalities in Pure and Applied Mathematics, vol. 4, article 73, no. 4, 2003.

[4] Z. Pavić, "Convex combinations, barycenters and convex functions," Journal of Inequalities and Applications, vol. 2013, article 61, 2013.

[5] C. P. Niculescu and C. I. Spiridon, "New Jensen-type inequalities," http://arxiv.org/abs/1207.6877.

[6] M. S. Moslehian and M. Kian, "Jensen type inequalities for Qclass functions," Bulletin of the Australian Mathematical Society, vol. 85, no. 1, pp. 128-142, 2012.

[7] Z. Pavić, J. Pečarić, and I. Perić, "Integral, discrete and functional variants of Jensen's inequality," Journal of Mathematical Inequalities, vol. 5, no. 2, pp. 253-264, 2011.

[8] J. Mićić, Z. Pavić, and J. Pečarić, “The inequalities for quasiarithmetic means," Abstract and Applied Analysis, vol. 2012, Article ID 203145, 25 pages, 2012.

[9] C. P. Niculescu and L. Persson, "Old and new on the HermiteHADamard inequality," Real Analysis Exchange, vol. 29, no. 2, pp. 663-685, 2003.

[10] S. S. Dragomir and C. E. M. Pearce, Selected Topics on HermiteHadamard Inequalities and Applications, RGMIA Monographs, Victoria University, Melbourne, Australia, 2000. 


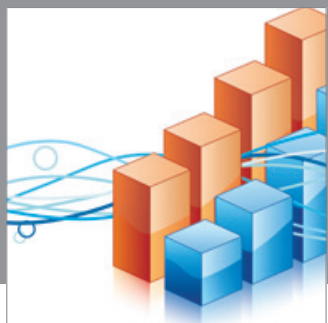

Advances in

Operations Research

mansans

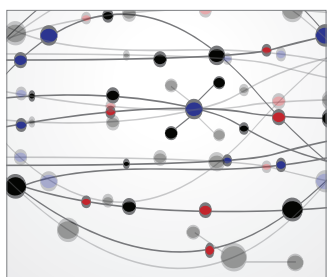

The Scientific World Journal
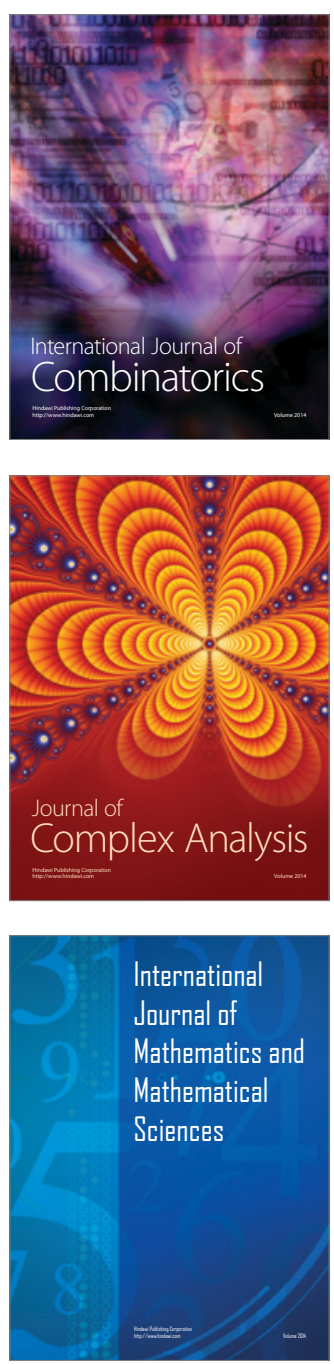
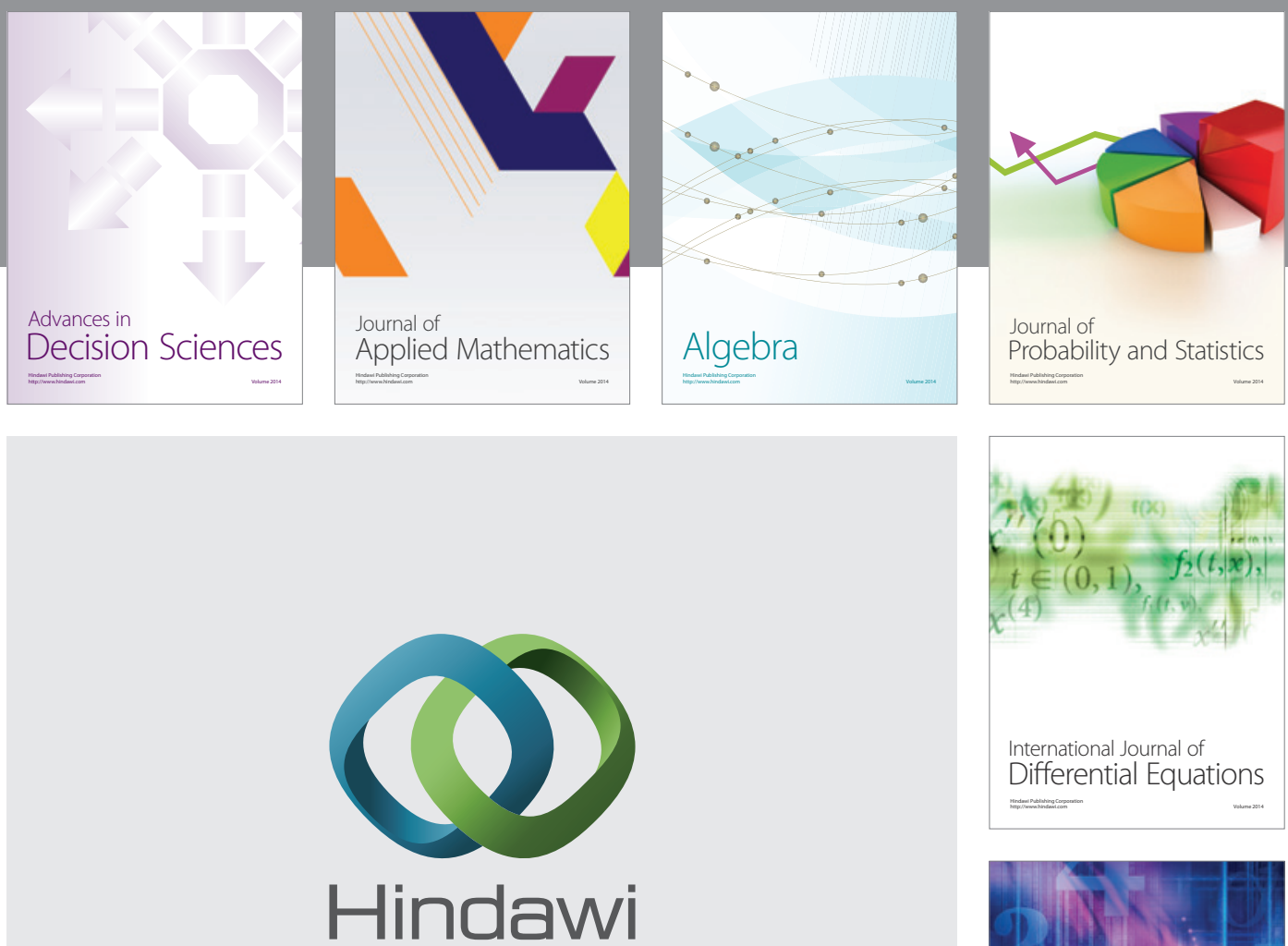

Submit your manuscripts at http://www.hindawi.com
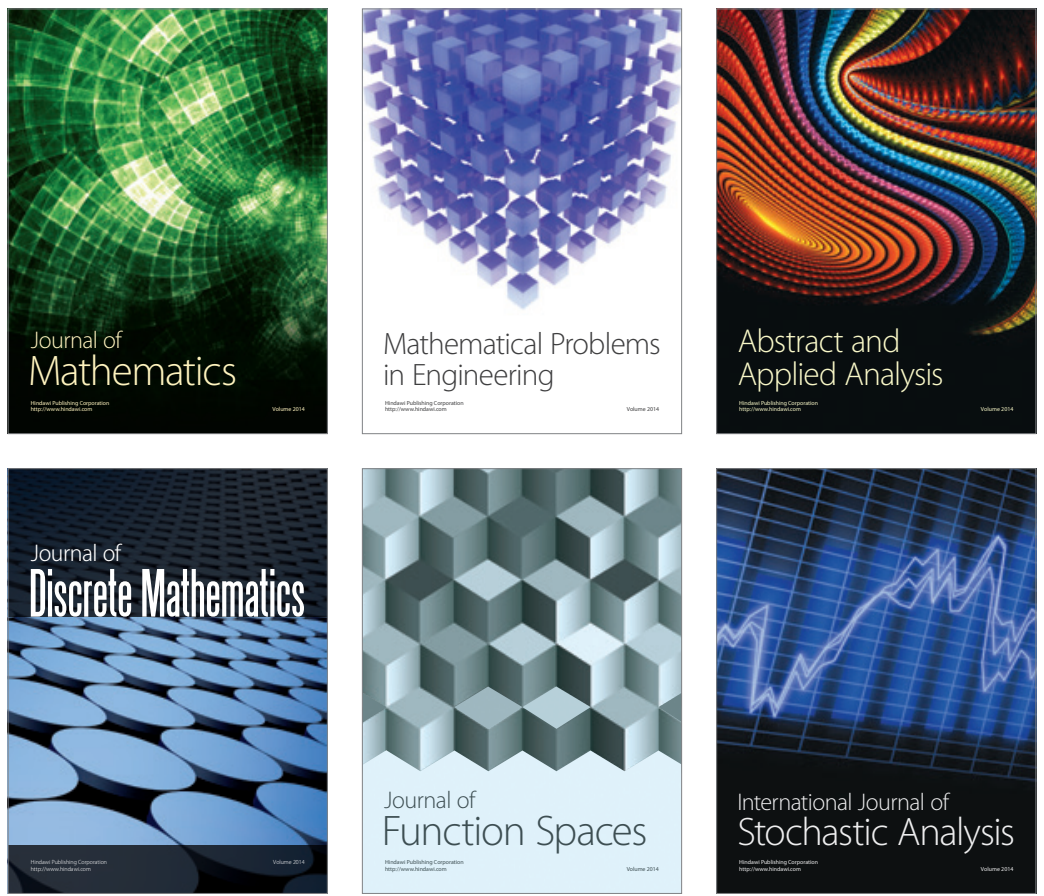

Journal of

Function Spaces

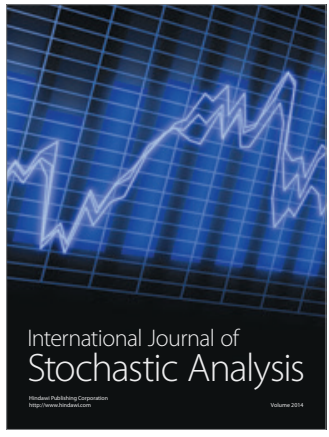

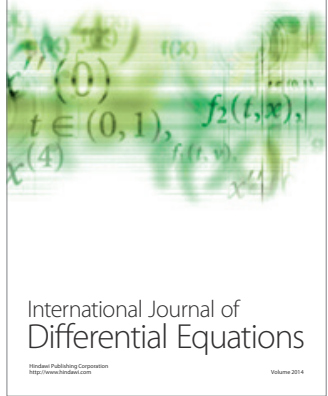
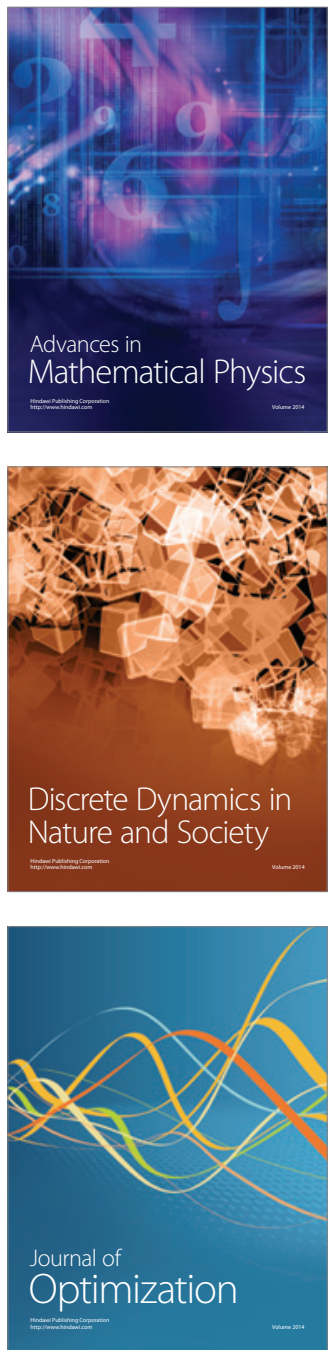